Rev SINAPSIS, Vol. 8, Nº 1, Junio 2016

ISSN $1390-7832$

\title{
Importancia de las tecnologías de la información y comunicación en la primera infancia
}

\section{Las tecnologías de la información en la infancia}

Ing. Enrique Javier Macías Arias. ${ }^{(1)}$

Lic. Blanca Celina Meza Cruz Mg. Pp ${ }^{(2)}$

Ing. Sandy Raúl Chun Molina Mg. Sc. ${ }^{(3)}$

Ing. Gregorio Isoldo Mendoza Cedeño Mg. Eds. ${ }^{(4)}$

(1) Instituto Tecnológico Superior Portoviejo, Ecuador

(2) Instituto Tecnológico Superior Paulo Emilio Macías, Ecuador

(3) Instituto Tecnológico Superior Paulo Emilio Macías, Ecuador

${ }^{(4)}$ Instituto Tecnológico Superior Paulo Emilio Macías, Ecuador

Contacto: enriquemacias21@ hotmail.com

Receptado: 10/03/2016 Aceptado: 16/05/2016

\section{Resumen}

La tecnología avanza notablemente en el mundo, las tecnologías de la información y comunicación (tics) han causado revuelo en la primera infancia, los niños de hoy tienen un alto grado en el manejo de la tecnología pero es preciso direccionarlos en un buen camino. Los niños se consideran nativos naturaleza de la tecnología pero debe ser acompañado pues trae consigo necesidad de conocimiento y en ocasiones se puede utilizar de forma inadecuada, es por esto que se debe capacitar en las tics, ya que hacen parte de la vida. Las tics cobran la mayoría de la parte del tiempo en infantes y adultos, sobre todo en los juegos pero es necesario darles tiempo para explorar y en compañía de sus padres se puede lograr una mejor comprensión del mundo del internet y las Tics, como consecuencia de la necesidad de información en la actualidad se han originado por parte de los docentes un interés por beneficiar a niños y niñas con el uso continuo de las Tics, donde los padres se hagan participes de dicho proceso y esto contribuya a mejorar no solo la parte académica de los estudiantes sino que al mismo tiempo se cree una cultura de pensamiento dirigida al mundo

Las tecnologías de la información en la infancia 
Rev SINAPSIS, Vol. 8, N 1, Junio 2016

ISSN $1390-7832$

de la internet donde se aborden competencias que se puedan desarrollarse con la integración y acceso a las tics desde la primera infancia.

Palabras claves: Niños, enseñanza, competencia, información

\title{
The technologies of information and communication in early childhood
}

\begin{abstract}
Technology advances greatly in the world, technologies of information and communication technologies (tics) have caused a stir in early childhood, children today have a high degree in technology management but we must address them in a good way. Children are considered native nature of technology but should be accompanied it brings need for knowledge and sometimes can be used improperly, is why should be trained in tics, and that are part of life. The tics charge most of the time in infants and adults, especially in games but you need to give them time to explore and accompanied by their parents can gain a better understanding of the world of Internet and tics, as a result of the need for information today have originated by teachers an interest benefit children with continued use of tics, where parents become participants in this process and this will help to improve not only academics students but at the same time a culture of thinking led to the world of the internet where skills that can be developed with integration and access to early childhood tics from addressing believed.
\end{abstract}

Keywords: Niños, enseñanza, competencia, información

\section{Introducción}

Se conoce como primera infancia, a la etapa del desarrollo de un infante que va desde la gestación hasta los 6 años. Durante este período de tiempo los niños y niñas deben recibir un cuidado especial, al igual que unos estímulos en cada dimensión que conforma su integridad.

Hoy en día se pueden dar cuenta que los niños desde que nacen están expuestos a múltiples estímulos sensoriales que años atrás no los percibían sino hasta

\section{Las tecnologías de la información en la infancia}


Rev SINAPSIS, Vol. 8, Nº 1, Junio 2016

ISSN $1390-7832$

que cumplían unos 5 o 6 años de edad, haciendo referencia a toda esta tecnología que ha venido incorporándose en nuestra cotidianidad y con la cual estamos complementando la crianza de nuestros hijos.

Ahora lo que podemos decir será bueno o malo que los niños a temprana edad tengan relación directa con todas las TICS.

Según varios autores tienen posiciones bastantes entusiastas en cuanto al uso de las TICS en la primera infancia, cada uno de ellos argumenta, pues el uso de estas nos lleva a tener que recapacitar y considerar que existen un anverso y un reverso de las tics, siendo conscientes que esta nueva tecnología día tras día está más presente tanto en nuestros hogares como en el aula de clase.

Entre los niños y las computadoras ya hay un amor y una estrecha relación entre estos dos, que los niños saben desde que nacen que ellos pertenecen a una nueva cultura digital, y de como ellos con facilidad las van asimilando y van haciendo uso de estas con espontaneidad. (Papert, 1997)

Las nuevas generaciones se han visto alteradas notoriamente, por el incremento de las tecnologías aunque no solo es debido a esto ya que hay diversos factores que están en nuestro contexto. El uso de estas tecnologías en el jardín infantil, se han vuelto unas aliadas que van en busca del mejoramiento de la calidad educativa. (Gros Salvat, 2004),

Ya que por medio de las tics, se generan nuevos aprendizajes siendo estos significativos para los niños, y contando can la ayudan del Internet se abren nuevas puertas al conocimiento ya que descubren, experimenta, y crean nuevos proyectos.

Desde que los niños nacen hasta los ocho años de edad, ellos aprenden de una forma más rápida percibiendo todo su contexto por medio de los sentidos, es por eso que las tecnologías multimedias pueden enriquecer estas experiencias, además de formar y

Las tecnologías de la información en la infancia

www.itsup.edu.ec/myjournal 
Rev SINAPSIS, Vol. 8, № 1, Junio 2016

ISSN $1390-7832$

contribuir a que todo su desarrollo cognitivo, social, personal, matemático y cultura universal. Lo importante es que se les enseñe de una forma responsable el contacto con estas tecnologías. (Vanscoter, 2001)

En los años 1960 y 1970 el término frecuente era tecnología informática, pero actualmente ha sido sustituida por tecnologías de la información y la comunicación (TIC). En un informe de la UNESCO se plantea que "las TIC se refieren a las tecnologías que se usan para procesar la información y la comunicación.” (UNESCO, 20012).

Debido a la integración de equipos con sistemas de comunicación, incluidas las tecnologías de audio y video, también se utilizan términos tales como multimedios o medios digitales. El pasaje de la tecnología informática del procesamiento de la información también hacia la comunicación aumenta su potencial para la educación.

En un informe de la UNESCO se sugiere que la introducción de las computadoras en la vida cotidiana, así como diferentes formas de las tecnologías digitales (como tablets, cámaras, televisores, teléfonos inteligentes, robots), requieren investigadores, responsables políticos y profesionales para reconsiderar el potencial de las TIC para la educación. (UNESCO, 20012)

Siguiendo a Castells, Internet es una red de redes de ordenadores capaces de comunicarse entre ellos... es mucho más que una tecnología. Es un medio de comunicación, de interacción y de organización social. "Significa que Internet es el medio en el que se basa una nueva forma de sociedad en la que vivimos que es la que el autor pasa a llamar sociedad red. „Sociedad cuya estructura social está construida en torno a redes de información a partir de la tecnología de información microelectrónica estructurada en Internet” (Castells, 2002).

Para historizar el concepto de Internet es necesario tener en cuenta que surge de una interacción entre la investigación universitaria, la ciencia, movimientos libertarios y programas de investigación militar. Internet se desarrolla desde el comienzo en base a una

\section{Las tecnologías de la información en la infancia}


Rev SINAPSIS, Vol. 8, № 1, Junio 2016

ISSN $1390-7832$

arquitectura abierta lo que permite que los usuarios aprovechen este libre acceso y produzcan también conocimiento, dando un constante feedback.

En el informe de la UNESCO de 2011, se plantea una "sociedad digital" en la que la información y la comunicación son conceptos básicos: la información se ha convertido en un bien económico valioso que se puede comprar y vender. Se desarrollan competencias y herramientas que un ciudadano de la sociedad digital debe dominar.

La educación es un derecho y permite el desarrollo como personas, hace posible el goce de otros derechos y el pleno ejercicio de la ciudadanía; difícilmente se podrá acceder a un empleo digno, ejercer la libertad de expresión o de participación, si no se tiene educación. Por consiguiente, destacamos la importancia de la educación y hacemos énfasis en ella desde la primera infancia y en todos los ámbitos, ya Utilización de tecnologías de la información y comunicación por niños y niñas, en ámbitos formales e informales que muchas veces se relaciona solamente con lo formal y con el comienzo de la educación primaria.

\section{Materiales y Métodos}

En cuanto al tipo de exploración que se utilizó en el proyecto es la; investigación de campo, que se apoya en informaciones donde se originan encuestas, mediante este tipo de investigación se utiliza el método de análisis descriptivo, se logra caracterizar un objeto de estudio o una situación concreta, señalar sus características y propiedades. La recolección de datos se efectuó por medio de encuestas que fueron aplicadas a 50 familias de la carrera de Desarrollo Infantil Integral del Instituto Tecnológico Superior Paulo Emilio Macías, que se encuentran en edades de 0 a 5 años. Se recopiló dicha información necesarias para realizar el diagnóstico del trabajo investigativo.

\section{Resultados}

En este apartado me centraré en el desarrollo global sobre el conjunto de la muestra objeto de investigación, que quedó configurada por 50 familias (50 encuestas), lo que supone un

\section{Las tecnologías de la información en la infancia}


Rev SINAPSIS, Vol. 8, № 1, Junio 2016

ISSN $1390-7832$

$71 \%$ del total de familias de la carrera de Desarrollo Infantil Integral existentes en el ITSPEM. Esto quiere decir que el índice de respuesta obtenido ha sido representativo.

El análisis de los resultados nos indica una participación mayoritaria de sexo femenino con un 95\%, seguido de un 5\% de participación masculina. Este dato ofrece información de la composición de la plantilla de Educación Infantil.

La composición de la muestra está formada por un $90 \%$ de estudiantes vinculadas al Ministerio de Inclusión Social (MIES) y un 10\% de estudiantes que no se encuentran vinculadas.

Los encuestados presentan un porcentaje muy alto en el número de computadoras como herramientas de enseñanza. Sólo un $2 \%$ destaca que no cuenta con ningún computador para su trabajo.

Un porcentaje alto de los encuestados destacan la poca integración de las TICs en sus Centros, seguido de la opinión de otro grupo de encuestados que piensa que hay bastante integración.

Un número alto de estudiantes opinan que en la educación infantil integra poco las TICs en su trabajo, seguido de la opción de un grupo de encuestados que valora esta integración como bastante.

Un número alto de encuestados destacan como bastante la importancia que las familias dan a la integración de las TICs en las aulas, lo que apoya sus propias iniciativas entorno a este tema.

\section{Discusión}

"La inteligencia digital tiene su raíz en un proceso de decisión, previo a todo lenguaje formalizado, que llamamos -opción clic-. Esta capacidad de decisión, de optar por sí o por no, es previa a las computadoras y al número." (Batro, 2003)

En otras palabras, la educación debe ser de calidad y debe darse ,a través de aprendizajes socialmente relevantes y experiencias educativas pertinentes a las necesidades y características de los individuos y de los contextos en los que se

\section{Las tecnologías de la información en la infancia}


Rev SINAPSIS, Vol. 8, № 1, Junio 2016

ISSN $1390-7832$

desenvuelven; El niño tiende a construir sentidos que se van atribuyendo desde los ámbitos familiares y escolares, y a través de estos y de los vínculos va transitando por un proceso de autoconstrucción. Como plantea Angeríz: „la educación cumplirá su papel en la determinación de los que será válido o prohibido, en la producción del sujeto social, dejando inscripciones sobre el sujeto individual... la educación produce en los sujetos la posibilidad de reconocerse como pertenecientes a un medio. De esta manera, continuamos enfatizando la importancia de la educación desde la primera infancia, y la encontramos ligada a los ámbitos formales e informales a través de los cuales el niño va construyendo su singularidad y su ser social. (Blanco Guijarro, 2011)

Para entender esta construcción desde lo escolar y lo familiar, la autora Dabas expone que „ambas organizaciones forman parte de un sistema social y cultural que significan funciones, adjudican posiciones y definen territorios de influencia y actuación, condicionando el accionar

Interés y motivación: Los niños se motivan al utilizar las TIC, esto hace que hace que el niño dedique con entusiasmo más tiempo al estudio $\mathrm{y}$, por tanto, es probable que aprenda mayor facilidad.

Interacción y actividad continua: Los niños se mantienen de manera constante en actividad intelectual y además pueden estar en comunicación con los demás, lo que les permite intercambiar experiencias y conocimientos sobre un tema, aspecto que representará la construcción del aprendizaje de manera más sólida y significativa.

Gran diversidad de información: El uso de las TIC en los procesos de aprendizaje da la oportunidad a los niños y al docente de tener acceso a diversidad de información, esto permite que el aprendizaje no se limite a temas tratados sólo en los libros y que además, no pierda actualidad.

Las tecnologías de la información en la infancia

www.itsup.edu.ec/myjournal 
Rev SINAPSIS, Vol. 8, N 1, Junio 2016

ISSN $1390-7832$

Programación del aprendizaje: Los niños pueden trabajar a su propio ritmo, no existe presión para avanzar a la velocidad de los demás. Cada niño puede programar los tiempos que dedicará para estudiar y los horarios en los que lo hará.

Desarrollo de la iniciativa: La constante participación en actividades que requieren tomar decisiones para avanzar en el estudio, propicia el desarrollo de su iniciativa.

Desarrollo de la habilidad para la búsqueda y selección de información: Al realizar una búsqueda y obtener información, el niño adquiere la habilidad de buscar, discriminar y seleccionar sólo lo que necesita, o lo que le puede ayudar en su proceso de aprendizaje.

Aprendizaje a partir de los errores: La realimentación inmediata para sus ejercicios y prácticas, permite al niño conocer los errores en el momento en que se producen, lo cual ayuda para su corrección.

Aprendizaje cooperativo: Los instrumentos que proporcionan las TIC pueden apoyar el trabajo en grupo y el cultivo de actitudes sociales, el intercambio de ideas, la cooperación, etcétera.

Desarrollo de habilidades para el uso de la tecnología: Se obtienen capacidades y competencias para el manejo de las máquinas relacionadas con la electrónica, aspecto que da valor agregado a los procesos de enseñanza aprendizaje del niño del docente.

Interés y motivación: Los niños se motivan al utilizar las TIC, esto hace que hace que el niño dedique con entusiasmo más tiempo al estudio y, por tanto, es probable que aprenda mayor facilidad.

Interacción y actividad continua: Los niños se mantienen de manera constante en actividad intelectual y además pueden estar en comunicación con los demás, lo que les permite intercambiar experiencias y conocimientos sobre un tema, aspecto que representará la construcción del aprendizaje de manera más sólida y significativa.

Las tecnologías de la información en la infancia

www.itsup.edu.ec/myjournal 
Rev SINAPSIS, Vol. 8, No 1, Junio 2016

ISSN 1390 - 7832

Gran diversidad de información: El uso de las TIC en los procesos de aprendizaje da la oportunidad a los niños y al docente de tener acceso a diversidad de información, esto permite que el aprendizaje no se limite a temas tratados sólo en los libros y que además, no pierda actualidad.

Programación del aprendizaje: Los niños pueden trabajar a su propio ritmo, no existe presión para avanzar a la velocidad de los demás. Cada niño puede programar los tiempos que dedicará para estudiar y los horarios en los que lo hará.

Desarrollo de la iniciativa: La constante participación en actividades que requieren tomar decisiones para avanzar en el estudio, propicia el desarrollo de su iniciativa. Desarrollo de la habilidad para la búsqueda y selección de información: Al realizar una búsqueda y obtener información, el niño adquiere la habilidad de buscar, discriminar y seleccionar sólo lo que necesita, o lo que le puede ayudar en su proceso de aprendizaje.

Aprendizaje a partir de los errores: La realimentación inmediata para sus ejercicios y prácticas, permite al niño conocer los errores en el momento en que se producen, lo cual ayuda para su corrección.

Aprendizaje cooperativo: Los instrumentos que proporcionan las TIC pueden apoyar el trabajo en grupo y el cultivo de actitudes sociales, el intercambio de ideas, la cooperación, etcétera.

Desarrollo de habilidades para el uso de la tecnología: Se obtienen capacidades y competencias para el manejo de las máquinas relacionadas con la electrónica, aspecto que da valor agregado a los procesos de enseñanza aprendizaje del niño del docente.

\section{Conclusiones}

Las tecnologías de la información en la infancia

www.itsup.edu.ec/myjournal 
Rev SINAPSIS, Vol. 8, N 1, Junio 2016

ISSN $1390-7832$

Las tecnologías de la información y comunicación aumentan y evolucionan constantemente en la actualidad, haciendo que tengamos que reflexionar sobre sus características, ventajas y desventajas. En los diversos entornos en los que los niños están inmersos, se encuentran gran variedad de TIC que les proveen de herramientas que necesitarán en su vida cotidiana y en su futuro como adulto. Es por eso que se hace necesaria una revisión sobre las características de los usos de TIC en niños, permitiendo obtener mayor información sobre este tema.

Con respecto a cuándo se comienzan a usar las tecnologías, tanto en el ámbito informal como formal, encontramos que a la edad de 3 años ya los niños y niñas realizan actividades con TIC. Aunque la edad promedio sería de 4 años, es decir, la mayoría de las experiencias dirigidas a los más chicos están pensadas para esa edad. Por tanto, los niños y niñas acceden a las tecnologías de la comunicación desde edades muy tempranas y pueden aprovechar y desarrollar las competencias que las TIC potencian.

En lo relativo a los dispositivos o aplicaciones más usadas aparecen diferencias en los distintos ámbitos. En las escuelas hay una gran variedad de actividades que se realizan con las TIC, pero las aplicaciones que más se utilizan están relacionadas con la lectoescritura, la matemática, el arte (música y dibujo) y con la comunicación (chats, correos electrónicos, maestros remotos). Mientras que en los hogares, los dispositivos que más usan los niños y niñas son los celulares, los televisores y la computadora. Si tomamos en cuenta las actividades que realizan en la computadora, los más frecuentes son los juegos y los videos.

En conclusión, enfatizamos la importancia del uso de TIC por parte de niños pequeños, ya que las tecnologías están incluidas en su vida cotidiana y van a tener que apropiarse de ellas para desarrollar competencias que necesitarán en esta sociedad digital. A los 3 o 4 años ya comienzan a interesarse por las TIC y a querer usarlas, por lo que se abren interrogantes en cuanto a cómo potenciar el aprendizaje mediado por TIC en niñas y niños pequeños. En consecuencia, sería importante unir esfuerzos entre la familia y la escuela, para lograr que se produzca un mejor proceso educativo que incluya los aspectos que se favorecen en los

\section{Las tecnologías de la información en la infancia}

www.itsup.edu.ec/myjournal 
Rev SINAPSIS, Vol. 8, Nº 1, Junio 2016

ISSN $1390-7832$

ámbitos informales y se potencien con aquellos que hacen a los ámbitos formales, ya que los cambios tecnológicos se dan en ambos espacios a la par.

\section{Bibliografía}

Batro, A. (2003). El aprendizaje actual. Argentina.

Blanco Guijarro, R. (2011). Educación inclusiva. Latina y el Caribe: CEE Participación Educativa.

Castells, M. (2002). La dimensión cultural de Internet. FUOC e ICUB.

Gros Salvat, B. (2004). La construcción del conocimiento en la red: límites y posibilidades. Barcelona: Universidad de Barcelona.

Papert, S. (1997). La familia conectada. Buenos Aires: Emecé Editores.

Santos, J. \&. (s.f.). Las tics en la primera infancia. Valorización e integración en la educación inicial a través. Revista iberoamericana de educación.

UNESCO. (20012). COMPENDIO MUNDIAL DE LA EDUCACIÓN.

Vanscoter. (2001). Las TIC en la primera infancia.

Las tecnologías de la información en la infancia

www.itsup.edu.ec/myjournal 